\title{
Levantamento do custo do processo admissional de técnico de enfermagem de um hospital de ensino*
}

\author{
SURVEY ON THE COST OF THE ADMISSION PROCESS \\ OF NURSING TECHNICIANS IN A TEACHING HOSPITAL
}

\author{
LEVANTAMIENTO DEL COSTO DEL PROCESO ADMISIONAL DE \\ TÉCNICO DE ENFERMERÍA DE UN HOSPITAL DE ENSEÑANZA
}

\author{
Helena Isuku Horibe Okano ${ }^{1}$, Valéria Castilho ${ }^{2}$
}

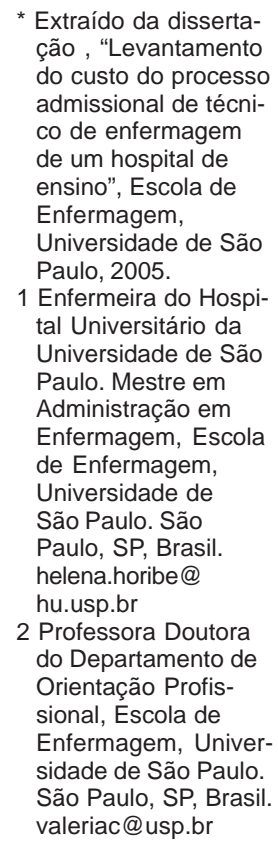

* Extraído da dissertação , "Levantamento do custo do processo admissional de técnico de enfermagem de um hospital de ensino", Escola de Enfermagem,

Universidade de São Paulo, 2005.

1 Enfermeira do Hospital Universitário da Universidade de São Paulo. Mestre em Administração em Enfermagem, Escola de Enfermagem, Universidade de São Paulo. São Paulo, SP, Brasil. helena.horibe@ hu.usp.br

2 Professora Doutora do Departamento de Orientação Profissional, Escola de Enfermagem, Universidade de São Paulo. São Paulo, SP, Brasil. valeriac@usp.br

\section{RESUMO}

Este estudo teve como objetivo contribuir para a gestão de custo do processo admissional de técnicos de enfermagem pelo mapeamento e mensuramento do custo direto das principais atividades desse processo. $\mathrm{O}$ estudo exploratório, retrospectivo, documental, na modalidade de estudo de caso foi realizado no Serviço de Apoio Educacional do Hospital Universitário da Universidade de São Paulo. O processo admissional foi dividido em cinco subprocessos: planejamento, recrutamento, seleção, contratação e treinamento admissional. Os resultados mostraram que o custo total direto do processo admissional foi de $\mathrm{R} \$$ $6.359,90$ e o subprocesso seleção foi o que mais consumiu recurso, com R \$3.416,40, representando $\mathrm{R} \$ 53,72 \%$ do total. Cada candidato contratado custou R $\$ 635,99$.

\section{DESCRITORES}

Pesquisa em administração de enfermagem.

Seleção de pessoal.

Recursos humanos de

enfermagem.

Custos e análise de custo.

\section{ABSTRACT}

This study was aimed at contributing to the cost management of the admission process of nursing technicians by mapping and measuring the direct cost of the main activities in this process. The exploratory, retrospective, documental study on the modality of case study was carried out at the Educational Support Service of the University of São Paulo's Hospital Universitário. The admission process was divided into five sub-processes: planning, recruiting, selection, hiring and admission training. Results showed that the direct total cost of the admission process was $\mathrm{R} \$ 6,359.90$, and that, within the sub-processes, selection was the one that consumed most resources - R \$ 3,416.40, amounting to $53.72 \%$ of the total. Each hired candidate cost R\$ 635.99.

\section{KEY WORDS}

Nursing administration research. Personnel selection.

Nursing staff.

Costs and cost analysis.

\section{RESUMEN}

Este estudio tiene como objetivo contribuir para la gestión de costo del proceso ad misional de técnicos de enfermería por el mapa y medición del costo directo de las principales actividades de ese proceso. El estudio exploratorio, retrospectivo, documental, en la modalidad de estudio de caso fue realizado en el Servicio de Apoyo Educacional del hospital Universitario de la Universidad de São Paulo. El proceso ad misional fue dividido en cinco subprocesos: planeamiento, reclutamiento, selección, contratación y entrenamiento ad misional. Los resul-tados muestran que el costo total directo del proceso ad misional fue de $\mathrm{R} \$ 6.359,90$, el sub-proceso de selección fue el que más consumió recurso con $\mathrm{R} \$ 3.416,40$ representando R \$ 53,72\% del total. Cada candidato contratado costó R $\$ 635,99$.

\section{DESCRIPTORES}

Investigatión em administración de enfermería.

Selección de personal.

Personal de enfermería.

Costos y análisis de costo. 


\section{INTRODUÇÃO}

A globalização da economia vem impulsionando as organizações a buscarem melhoria contínua, eficiência e eficácia nos processos de trabalho. Desse modo, constantemente, atualizam suas estratégias para sobreviverem no complexo ambiente social, em que ocorrem mudanças rápidas e drásticas nas áreas econômica, política, tecnológica, cultural e de mercado.

Essas mudanças geram aumento da competitividade, fato que tem levado as organizações a buscarem abordagens inovadoras, essenciais para sua existência e sobrevivência.

Nesse sentido, os profissionais que as integram, precisam desenvolver competências para trabalharem em uma perspectiva mais global e não reducionista, entendendo do negócio e das estratégias da organização, independente da área de atuação.

Em decorrência disso, outra competência necessária aos profissionais gestores, tanto da área industrial e comercial como de serviço é a gestão de custos, elemento importante para tomada de decisão de uma empresa ${ }^{(1)}$.

A mesma lógica aplica-se às organizações hospitalares que também vivenciam momentos de grandes mudanças e de competitividade que exigem a prestação de serviços de qualidade com baixo custo e resolutividade, obrigando-as a adotarem estratégias de excelência para garantir sua sobrevivência ${ }^{(2)}$.

Por isso, a importância do controle de custos nas instituições de saúde tem sido destacada, sejam elas privadas ou públicas, já que a demanda por serviços de saúde pela população é grande e os recursos $\operatorname{limitados}^{(3)}$.

Além disso, as inúmeras dificuldades de ordem econômico-financeiras, decorrentes de uma conjuntura nacional extremamente complexa, têm contribuído para tornar o custo tema essencial no gerenciamento das organizações hospitalares, tanto privadas como públicas, com fins lucrativos ou mesmo filantrópicos ${ }^{(4)}$.

Outro aspecto a ser destacado em relação à necessidade de gerenciamento dos custos na saúde, é que, de um modo geral, as instituições de saúde possuem muito desperdício de recursos.

Este resulta, diretamente, da inexistência de um sistema técnico de custos, havendo um desconhecimento e conseqüente despreocupação com eles. Neste aspecto, a identificação adequada dos custos, receitas e resultados, em relação a atividades, centros de custos e unidades de negócio cons- tituem-se em um primeiro passo importante para uma gestão de sucesso, direcionada a efetivos objetivos de saúde econômico-financeira da instituição. Em boa parte, o sucesso no gerenciamento dos custos depende da conscien-tização das áreas envolvidas, do apoio da direção e da mobilização de todos na busca de melhores indicadores de desempenho ${ }^{(4)}$.

Pautado no exposto, fica evidente que o controle de custo é imprescindível à sobrevivência das instituições de saúde, visto que as privadas dependem das receitas geradas na prestação de serviços e as públicas de orçamentos exíguos.

Em razão da escassez de recursos, as organizações hospitalares devem buscar ferramentas gerenciais que fundamentem e proporcionem melhoria nas tomadas de decisões para otimizá-las e garantir uma assistência contínua com qualidade.

Custos crescentes e recursos escassos têm afetado todos os prestadores de serviço de saúde e não houve outro momento em que as organizações de saúde tiveram de operar com mais eficiência ou ter mais cuidado com as restrições de custos, como na atualidade. A restrição de custos deve estar associada a uma oferta eficiente e eficaz de serviço, ao mesmo tempo em que gera a receita necessária, para que se dê continuidade à produtividade organizacional ${ }^{(5)}$.

O envolvimento e o comprometimento dos profissionais de saúde na área de análise de custo tornam-se imprescindíveis, para que as metas de qualidade assistencial sejam atendidas ${ }^{(6)}$.

A crescente elevação dos custos na saúde trouxe aos profissionais da área a necessidade de adquirir conhecimentos teóricos sobre o tema e, conseqüentemente, sua aplicação na realização de estudos, em que se busca a racionalização na alocação de recursos e a otimização de resultados ${ }^{(7)}$.

$\mathrm{Na}$ atualidade, as enfermeiras estão sendo envolvidas em decisões financeiras e no planejamento orçamentário das instituições onde trabalham, constituindo um nível decisório im-portante na alocação de recursos humanos, materiais e físicos, visto que estabelecem prioridades de serviços nas unidades de trabalho; decidem quem prestará os cuidados e quanto tempo será despendido para sua realização e quais recursos serão empregados. Portanto, essas decisões afetam diretamente o número e a qualidade dos atendimentos $\operatorname{prestados}^{(8)}$.

O desenvolvimento de estudos sobre custos é uma área relativamente nova às enfermeiras que têm descoberto a utilidade dessa ferramenta gerencial, permitindo, além de conhecimento do custo dos produtos e processos, a tomada de decisão em relação aos cortes e controle de desperdícios ${ }^{(9)}$. 
Em suma, cabe não só às enfermeiras, mas a todos os profissionais da área de saúde, o compromisso de buscar o equilíbrio entre qualidade, quantidade e custos utilizados na assistência, preservando a qualidade do atendimento e buscando novas soluções para seu gerenciamento ${ }^{(10)}$.

Nesta direção, optou-se por estudar o custo do processo admissional do Serviço de Apoio Educacional (SED) do Hospital Universitário da Universidade de São Paulo, local de trabalho da pesquisadora. Esse serviço tem como missão manter a equipe de enfermagem com elevado nível de qualificação pessoal, ética e técnica e compartilha com outros serviços a responsabilidade pelos processos de admissão, treinamento e avaliação do desempenho da equipe de enfermagem.

O processo admissional tem a finalidade de selecionar pessoa no mercado de trabalho que correspondam ao perfil que a organização deseja e valoriza, e compreende o recrutamento, a seleção, o treinamento e a contratação.

Vale ressaltar que o processo admissional, sobretudo, nas instituições públicas, em razão da especificidade da área de enfermagem, da legislação vigente e da política de Recursos Humanos, entre outras, tem se revelado extremamente complexo, envolvendo inúmeros recursos.

A busca na literatura por estudos sobre o custo de um processo de recrutamento, seleção e treinamento admissional do pessoal de enfermagem demonstrou a inexistência de referências específicas a respeito do assunto. Em estudo específico sobre as publicações de custo em enfermagem no período de 1966 a 2000, também, não foram encontrados trabalhos publicados sobre o tema ${ }^{(11)}$.

Diante do exposto, optou-se pelo desenvolvimento do estudo do custo do processo admissional de Técnico de Enfermagem do Hospital Universitário da Universidade de São Paulo (HU-USP). Acredita-se que este estudo, somado a outros, poderá fornecer informações detalhadas sobre o custo do gerenciamento de pessoal de enfermagem no HUUSP, favorecendo a otimização da condução do processo admissional desses e de outros profissionais.

\section{OBJETIVO}

Este estudo teve como objetivo contribuir para a gestão de custo do processo admissional de técnicos de enfermagem pelo mapeamento e mensuração do custo direto das principais atividades desse processo.

\section{MÉTODO}

Trata-se de um estudo exploratório na modalidade estudo de caso onde se analisou o custo direto das principais atividades do processo admissional de técnico de enfermagem do HU-USP, ocorrido no período de setembro a novembro de 2003.
O processo admissional foi aberto para o preenchimento de dez vagas e contou com 192 inscritos, sendo que 176 compareceram para a prova escrita. Desses, foram aprovados na prova escrita 74 candidatos. Na prova prática foram selecionados e classificados 49 candidatos entre os 74 , sendo contratados os dez primeiros classificados. Os demais ficaram aguardando a convocação, à medida que surgirem novas vagas. Essa lista tem validade por um ano, podendo ser prorrogada.

A pesquisa foi avaliada e aprovada pela Comissão de Ensino e Pesquisa do HU-USP.

Para a coleta de dados foram elaboradas duas planilhas: a planilha de registro de atividades do processo e a planilha de cálculo de custos de atividades do processo. A primeira constou da descrição das principais de atividades do processo, dos profissionais envolvidos, do tempo total despendido e do material gasto. A segunda foi composta pelas atividades, os profissionais envolvidos; o tempo unitário e total (minutos) despendido pelos recursos humanos; custo unitário e custo total da mão-de-obra direta (MOD) e custo do material.

O preenchimento dos dados da planilha de registro de atividades do processo, com as etapas, o tempo do pessoal envolvido e o levantamento dos recursos materiais foi realizado pela pesquisadora, ao acompanhar o processo admissional de técnico de enfermagem, no período de setembro a novembro de 2003. O tempo gasto pelos profissionais, nas diferentes atividades, foi cronometrado pela pesquisadora.

Em relação ao tempo gasto para elaboração dos exames admissionais pelo Serviço de Laboratório Clínico (SLC) e Serviço de Iconologia (SI) a pesquisadora cronometrou 5 procedimentos de cada tipo e calculou a média.

Para o levantamento do tempo da consulta médica foi utilizado os registros das consultas do Serviço Médico e, também, foi calculada a média de tempo utilizado

Para calcular o custo da MOD por minuto dos profissionais envolvidos, foi usada a Tabela de salário vigente em agosto de 2004, disponível no site da Universidade (www.recad.usp.br), uma vez que não houve alteração de salário no período estudado. Para o cálculo foram considerados o salário inicial mais o adicional de insalubridade pago à maioria dos profissionais que trabalham no hospital com exceção dos docentes, secretária e técnico de Recursos Humanos. A estes valores, foi acrescida a verba de representação para alguns profissionais de enfermagem. Valores estes obtidos também no site acima citado. Ao salário bruto foram acrescidos os encargos sociais.

Para o cálculo de custo foi considerada a jornada de trabalho de 180 horas para os profissionais do Departamen- 
to de Enfermagem (DE), SLC e SI; 240 horas para docentes da Escola de Enfermagem da USP, secretária do SEd e técnico de Recursos Humanos do Serviço de Pessoal e 120 horas para o médico do trabalho.

Assim, o custo da mão de obra de cada profissional foi calculada pelo custo da MOD por minuto multiplicado pelo tempo gasto na atividade em minutos.

Para o levantamento do custo de material consumido foram utilizados os registros do Serviço de Compras (SC) e calculada a média dos preços das compras realizadas.

O custo total do processo foi calculado pelo somatório do custo de pessoal e material de cada subprocesso.

O custo unitário do candidato selecionado foi o somatório dos subprocessos planejamento, recrutamento e seleção divididos pelo número de candidatos selecionados (49).

Para o cálculo do custo unitário do candidato contratado foi o somatório dos cinco processos divididos pelo número de candidatos contratados (10).

Em razão do fato do HU-USP não contar com um sistema de custo que possibilite a aferição mais acurada dos recursos envolvidos no processo admissional, ou seja, os custos diretos e indiretos, foram custeados apenas os custos diretos (materiais e recursos humanos).

Por custos diretos entendem-se aqueles que são diretamente apropriados a um objeto de custo, bastando haver uma medida objetiva de consumo. Já os custos indiretos são aqueles que não podem ser diretamente apropriados a um objeto de custo, senão por meio de rateios estimados ou $\operatorname{arbitrários~}^{(12)}$.

Para efeito do cálculo do processo admissional, não foram considerados os custos diretos dos materiais utilizados nos exames laboratoriais e no exame radiológico, pois estes serviços não possuem o custo de seus procedimentos.

Assim, o custo do processo apresentado corresponde a uma estimativa, não havendo precisão na aferição e na análise do custo total.

Os dados obtidos resultaram em figuras e tabelas em que foram expostas as atribuições das parcelas de recursos às atividades e os custos.

\section{APRESENTAÇÃOE DISCUSSÃO DOS RESULTADOS}

\section{Mapeamento do processo}

Pela complexidade de suas ações, o processo admissional foi dividido em cinco subprocessos: planejamento, re- crutamento, seleção, contratação e treinamento admissional, conforme demonstrado no desenho do fluxograma (Anexo I).

Cada subprocesso apresenta as seguintes atividades, descritas a seguir:

$\Rightarrow$ Planejamento: indicar a banca examinadora, elaborar edital, elaborar prova escrita, digitar prova escrita, elaborar prova prática e digitar prova prática;

$\Rightarrow$ Recrutamento: publicar o edital, confeccionar cartazes e realizar a inscrição;

$\Rightarrow$ Seleção: reproduzir prova escrita, conferir documentos dos candidatos, aplicar a prova escrita, corrigir prova escrita, listar os candidatos aprovados, digitar a lista dos candidatos aprovados, divulgar a lista dos aprovados, reproduzir a prova prática, preparar a estação para prova prática, realizar prova prática, classificar os candidatos aprovados, digitar a lista de candidatos classificados e publicar a classificação dos candidatos.

$\Rightarrow$ Contratação: convocar candidato, orientar o candidato, receber documentação do candidato, realizar exames laboratoriais, realizar radiografia, agendar exame médico, realizar exame médico e realizar contratação do candidato.

$\Rightarrow$ Treinamento Admissional: elaborar o programa, reproduzir o material de treinamento, ministrar conteúdo teórico, revisar a prática de procedimentos de enfermagem, revisar a prática de diluição e preparo de medicamento, realizar avaliação do funcionário, montar prontuário funcional e encaminhar para unidade.

Após a descrição de cada atividade e cronometrados os tempos de cada uma em minutos foi elaborado um dicionário de atividades contendo as principais atividades, a função do executante e o tempo de execução de cada atividade, totalizando 38, conforme ilustrado na Figura 1.

Nota-se pela Figura 1 que as atividades que mais consumiram tempo, em minutos, foram: a aulas teóricas (300), elaboração do edital (255), publicação do edital (120) e correção da prova escrita (120). Em serviços públicos a elaboração de editais é um procedimento cuidadoso e complexo que requer muita atenção, uma vez que se houver alguma falha ou incoerência em seu conteúdo pode haver impugnação do concurso.

Os recursos humanos envolvidos no processo foram: 7 enfermeiros ( 2 docentes da Escola de Enfermagem da Universidade de São Paulo, 1diretor de serviço, 1 chefe de seção e 3 enfermeiros assitenciais), 3 funcionários administrativos (1 secretária do SEd e 2 técnicos de Recurso Humano do Serviço de Pessoal), 1 médico do trabalho, 3 funcionários do SLC (1 farmacêutico-bioquímico, 1 técnico de laboratório e 1 auxiliar de laboratório) e 1 técnico de radiologia. 


\begin{tabular}{|c|c|c|}
\hline Função & Atividades principais & Tempo (Minutos) \\
\hline $\boldsymbol{\varphi}$ & Indicar a banca examinadora & 10 \\
\hline 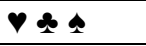 & Elaborar edital & 255 \\
\hline 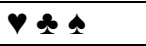 & Elaborar prova escrita & 50 \\
\hline$\bullet$ & Digitar prova escrita & 95 \\
\hline 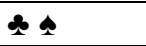 & Elaborar prova prática & 120 \\
\hline$\bullet$ & Digitar prova prática & 35 \\
\hline$\Delta$ & Publicar o edital & 120 \\
\hline$\bullet$ & Confeccionar Cartazes & 20 \\
\hline$\Delta$ & Realizar a inscrição dos candidatos & 3 \\
\hline$\bullet$ & Reproduzir a prova escrita & 2 \\
\hline - 1 & Conferir documentos dos candidatos & 1 \\
\hline s & Aplicar a prova escrita & 120 \\
\hline$P$ Q & Corrigir prova escrita & 9 \\
\hline s & Classificar e listar os candidatos aprovados na prova escrita & 0,5 \\
\hline$\bullet$ & Digitar a lista de candidatos aprovados & 15 \\
\hline$\bullet$ & Divulgar lista dos aprovados & 10 \\
\hline$\bullet$ & Reproduzir a prova prática & 0,3 \\
\hline s & Preparar a estação para prova prática & 12 \\
\hline 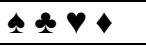 & Aplicar prova prática & 30 \\
\hline 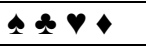 & Classificar os candidatos aprovados na prova prática & 1 \\
\hline$\bullet$ & Digitar a lista de candidatos classificados & 5 \\
\hline$\Delta$ & Digitar e enviar por e-mail a lista dos classificados para publicação & 10 \\
\hline$\Delta$ & Convocar candidato & 3 \\
\hline$\Delta$ & Orientar o candidato & 3 \\
\hline $\mathbf{\square} \circ \square$ & Realizar exames laboratoriais & 37 \\
\hline$\diamond$ & Realizar exame radiológico & 10 \\
\hline$\Delta$ & Agendar exame médico & 2 \\
\hline$\Delta$ & Realizar exame médico & 30 \\
\hline$\Delta$ & Receber documentação dos candidatos & 3 \\
\hline$\Delta$ & Realizar contratação do candidato & 25 \\
\hline s & Elaborar o programa & 40 \\
\hline$\bullet$ & Reproduzir o material de treinamento & 3 \\
\hline s & Ministrar conteúdo teórico & 300 \\
\hline s & Revisar as técnicas de procedimentos & 22 \\
\hline$\underline{1}$ & Revisar as técnicas de administração e diluição de medicamentos & 25 \\
\hline s & Realizar avaliação do funcionário & 10 \\
\hline$\bullet$ & Montar prontuário funcional & 5 \\
\hline s & Encaminhar para unidade & 5 \\
\hline
\end{tabular}

Figura 1 - Dicionário de atividades descritas nos subprocessos - São Paulo - 2005.

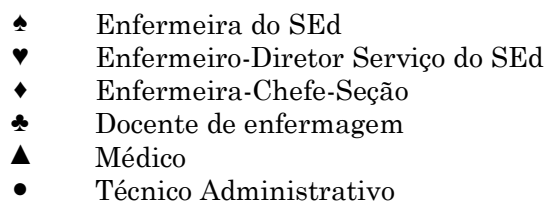

Cabe esclarecer que esses profissionais participaram de diferentes atividades em cada subprocesso, descritas anteriormente, dedicando tempos diferentes. Assim, na Tabela 1 apresentamos apenas o resumo do tempo gasto pelas equipes no processo todo. 
Tabela 1 - Resumo do tempo gasto (minuto) pelas equipes no processo admissional de técnicos de enfermagem São Paulo - 2005

\begin{tabular}{|c|c|c|c|c|c|c|}
\hline $\begin{array}{c}\text { Subprocessos } \\
\text { Recursos }\end{array}$ & Planejamento & Recrutamento & Seleção & Contratação & $\begin{array}{l}\text { Treinamento } \\
\text { Admissional }\end{array}$ & Total \\
\hline Enfermeiros & 2.710 & - & 7.089 & - & 2.150 & 11.949 \\
\hline Administrativo & 130 & 716 & 504,2 & 360 & 80 & $1.790,2$ \\
\hline Médico & - & - & - & 300 & - & 300 \\
\hline $\mathrm{S} \mathrm{L} \mathrm{C}$ & - & - & - & 370 & - & 370 \\
\hline S I & - & - & - & 100 & - & 100 \\
\hline Total & 2.840 & 716 & $7.593,2$ & 1.130 & 2.230 & $14.509,2$ \\
\hline
\end{tabular}

O total de tempo despendido pelas equipes no processo foi de 14.509,2 minutos que correspondem aproximadamente 242 horas de trabalho.

A seleção foi o subprocesso que mais consumiu tempo dos Recursos Humanos, com 7.593,2 minutos (52,33\%), seguido do planejamento com 2.840 minutos $(19,57 \%)$, treinamento admissional com 2.230 minutos (15,37\%), contratação com 1.130 minutos $(7,79 \%)$ e recrutamento com 716 minutos $(4,93 \%)$.

Neste processo, a equipe de enfermeiros dedicou $82,35 \%$ do total de horas despendidas, e os demais profissionais dedicaram $17,65 \%$. Vale lembrar que este processo estudado é o de técnico de enfermagem, portanto, a atuação dos enfermeiros, no processo, é importante na escolha do profissional com quem irá trabalhar.

\section{Custo com pessoal}

Conforme demonstrado nos dados da Tabela 2, o custo total de Recursos Humanos foi de R $\$ 5.351,36$ e o custo de enfermeiros foi de $\mathrm{R} \$ 4.836,03$, o que corresponde a $90,37 \%$ do total.

Tabela 2 - Resumo dos custos diretos de Recurso Humano envolvidos nos subprocessos do processo admissional de técnicos de enfermagem - São Paulo - 2005

\begin{tabular}{lcccccr}
\hline $\begin{array}{l}\text { Processos } \\
\text { Recursos }\end{array}$ & Planejamento & Recrutamento & Seleção & Contratação & $\begin{array}{c}\text { Treinamento } \\
\text { Admissional }\end{array}$ & Total \\
\hline Enfermeiros & $1.124,55$ & - & $2.915,98$ & - & 795,50 & $4.836,03$ \\
Administrativo & 18,20 & 100,24 & 70,59 & 50,40 & 11,20 & 250,63 \\
Médico & - & - & - & 168,00 & - & 168,00 \\
S L C & - & - & - & 77,70 & - & 77,70 \\
S I & - & 100,24 & $2.986,57$ & 315,10 & 806,70 & $5.351,36$ \\
\hline Total & $1.142,75$ & & &
\end{tabular}

\section{Custo total direto do processo admissional}

Os dados da Tabela 3 mostram o resumo dos custos

diretos de Recursos Humanos e materiais envolvidos em cada subprocesso do processo admissional.

Tabela 3 - Resumo dos custos diretos de Recursos Humanos e materiais - São Paulo - 2005

\begin{tabular}{lccrr}
\hline \multicolumn{1}{c}{ Subprocesso } & $\begin{array}{c}\text { Custo com } \\
\text { Pessoal }\end{array}$ & Custo com Material & Custo Total & $\%$ \\
\hline Planejamento & $1.142,75$ & - & $1.142,75$ & 17,97 \\
Recrutamento & 100,24 & 473,30 & 573,54 & 9,02 \\
Seleção & $2.986,57$ & 429,83 & $3.416,40$ & 53,72 \\
Contratação & 315,10 & - & 315,10 & 4,95 \\
Treinamento admissional & 806,70 & 105,41 & 912,11 & 14,34 \\
\hline \multicolumn{1}{c}{ Total } & $5.351,36$ & $1.008,54$ & $6.359,90$ & 100,00 \\
\hline
\end{tabular}

O custo total direto do processo admissional de técnico de enfermagem foi de $\mathrm{R} \$ 6.359,90$, desses, $\mathrm{R} \$ 5.351,36$ $(84,14 \%)$ foram gastos com pessoal e $\mathrm{R} \$ 1.008,90(15,86 \%)$ com material.

O subprocesso seleção consumiu R \$3.416,40, (53,72\%), seguido do planejamento com R\$ 1.142,75 (17,97\%), treinamento admissional com R $\$ 912,11$ (14,34\%), recrutamento com $\mathrm{R} \$ 573,54$ (9,02\%) e contratação com $\mathrm{R} \$ 315,10$ (4,95\%).
O custo por candidato selecionado (planejamento + recrutamento + seleção) foi R $\$ 104,75$, e ele absorveu os custos dos candidatos não selecionados, lembrando que foram selecionados 49 candidatos.

O custo por funcionário contratado foi $\mathrm{R} \$ 635,99$, considerando os dez primeiros candidatos classificados e contratados, conforme disponibilidade das vagas existentes no momento da abertura do processo seletivo. 


\section{CONCLUSÃO}

- O processo admissional compreendeu cinco sub-processos interligados e complementares, sendo: o planejamento, o recrutamento, a seleção, a contratação e o treinamento admissional.

- O custo total direto do processo admissional foi $\mathrm{R} \$$ $6.359,90$, sendo que o custo com pessoal foi de $\mathrm{R} \$ 5.351,36$ representando $84,14 \%$ do custo total e do material foi $\mathrm{R} \$ 1.008,54$ correspondendo a $15,86 \%$ do custo total.

- $\mathrm{O}$ custo direto do subprocesso seleção foi $\mathrm{R} \$ 3.416,40$ respondendo a $53,72 \%$ do total gasto.

- custo de cada candidato selecionado foi estimado R\$ 104,75 e do funcionário contratado $\mathrm{R} \$ 635,99$.

\section{CONSIDERAÇÕES FINAIS}

Ao realizar este estudo, pretendeu-se contribuir para a gestão do custo do processo admissional de técnico de enfermagem no HU-USP por meio do mapeamento e mensuramento do custo direto das principais atividades do processo admissional desse profissional.

Cabe enfatizar que o HU-USP não conta com um sistema de custo que possibilite a aferição mais acurada dos recursos envolvidos nos processo admissional, de um modo geral, e neste caso especificamente de técnicos de enfermagem. Assim, o custo do processo apresentado corresponde a uma estimativa, não havendo precisão na aferição e na análise do custo total.

\section{REFERÊNCIAS}

1. Martins E. Contabilidade de custos. $5^{\text {a }}$ ed. rev. São Paulo: Atlas; 1996.

2. Cardoso S. Contribuição para o estudo dos custos unitários das análises laboratoriais e sua comparação com os preços estabelecidos pelo Sistema Único de Saúde - SUS em um laboratório hospitalar em 2001 [dissertação]. São Paulo: Faculdade de Ciências Farmacêuticas, Universidade de São Paulo; 2003.

3. Anselmi ML. Quadro de referência para elaboração do orçamento de enfermagem em instituições de saúde [tese livre-docência]. Ribeirão Preto: Escola de Enfermagem de Ribeirão Preto, Universidade de São Paulo; 2000.

4. Beulke R, Bertó DJ. Gestão de custos e resultados na saúde: hospitais, clínicas, laboratórios e congêneres. $2^{\mathrm{a}}$ ed. São Paulo: Saraiva; 2000.

5. Marquis BL, Huston CJ. Administração e liderança em enfermagem: teoria e aplicação. 2a ed. Porto Alegre: Artes Médicas; 1999.

6. Matos AJM. Retorno de investimento: uma avaliação da empresa hospitalar. Mundo Saúde. 1979;3(4):243-5.
No entanto, o mapeamento das atividades de cada subprocesso e o levantamento dos recursos em cada etapa do mesmo permitiu a visualização de como estão sendo consumidos. O que possibilita a realização de propostas para otimização dos recursos.

Exemplificando, os resultados evidenciados propiciam algumas reflexões sobre a forma de selecionar os candidatos. A avaliação da prova prática através da execução de procedimento de enfermagem teve um custo alto dentro do processo que poderia ser minimizado se fosse aplicada uma prova oral, conforme vêm sendo feito em outros hospitais. No entanto, há necessidade de antes estudar a eficácia de cada método.

Assim, acredita-se que este estudo possa trazer subsídios à equipe do Serviço de Apoio Educacional, para refletir sobre a atual forma de condução do processo admissional, sobretudo em relação à alocação de recursos, almejando mudanças que possam contribuir para redução dos custos sem comprometer a qualidade do processo.

A falta de estudos abordando a temática custo do processo admissional, não só de técnicos de enfermagem, mas também dos demais profissionais da área de saúde, dentro do próprio HU-USP como em outros locais, impede a comparação de valores.

No entanto, em relação ao conhecimento de custos em enfermagem, mais importante que os valores encontrados neste estudo, foi a proposição de um modelo de aferição de custos de um programa admissional, que pode ser adaptado e melhorado para outros serviços e revisado em novos estudos.

7. Francisco IMF, Castilho V. A enfermagem e o gerenciamento de custos. Rev Esc Enferm USP. 2002;36(3):240-4.

8. Consejo Internacional de Enfermeras (ICN). La calidad, los costos y la enfermera. Ginebra; 1993.

9. Ribeiro FG. Análise dos custos diretos no ato operatório da revascularização do miocárdio: insumos e categorias profissionais [dissertação]. São Paulo: Escola de Enfermagem, Universidade de São Paulo; 1998.

10. Bittar E. Consumo e custo médio direto do material utilizado em cirurgia de revascularização do miocárdio [dissertação]. São Paulo: Escola de Enfermagem, Universidade de São Paulo; 2001.

11. Oliveira JC. Estudo bibliométrico das publicações de custos em enfermagem no período de 1966 a 2000 [dissertação]. São Paulo: Escola de Enfermagem, Universidade de São Paulo; 2001.

12. Ching HY. Manual de custos de Instituições da Saúde: sistemas tradicionais de custos e sistemas de custeio baseado em atividades (ABC). São Paulo: Atlas; 2001. 


\section{ANEXO I}

Fluxograma do processo admissional

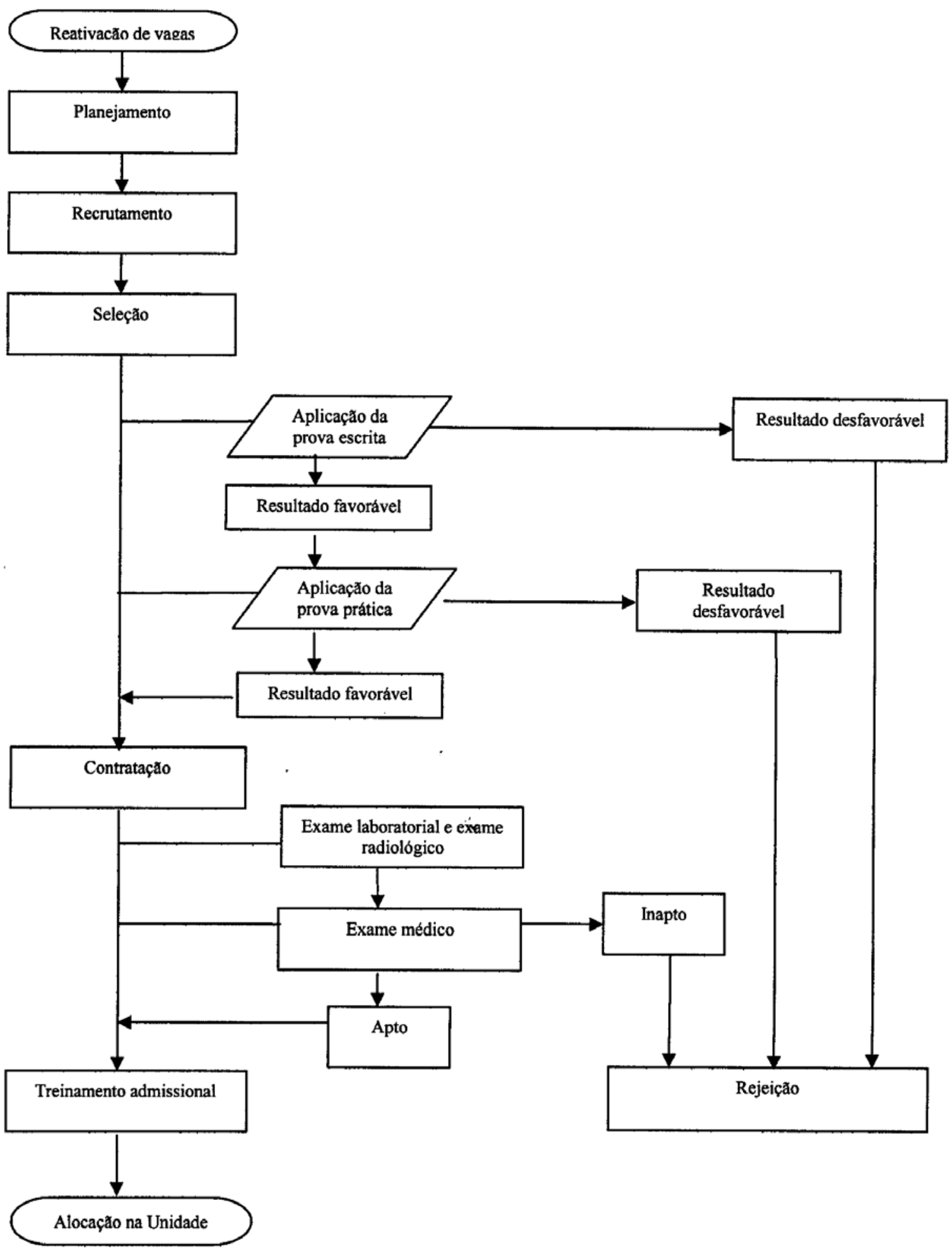

